\title{
Optical High-Capacity Satellite Downlinks via High-Altitude Platform Relays
}

\author{
Markus Knapek*, Joachim Horwath, Florian Moll, Bernd Epple, Nicolas Courville, Hermann Bischl, \\ Dirk Giggenbach
}

German Aerospace Center DLR, Oberpfaffenhofen, 82234 Wessling, Germany

\begin{abstract}
Earth-observation (EO) satellite missions produce a large amount of data using high-resolution optical or radar sensors. During the last decades the amount of data has steadily increased due to improved sensor technologies with increased temporal resolution, sensor resolution, and pixel count. As a consequence EO satellite missions have become limited by the downlink data rates of microwave communication systems, which are inhibited by spectrum restrictions, manageable antenna sizes, and available transmit power. Optical downlinks from EO satellites with data rates of several Gbps mitigate the limiting effects of microwave communication systems; however optical links do not provide the necessary link availability through the atmosphere due to cloud blockage above the ground station. Apart from diversity concepts with several ground stations or satellite networks, a stratospheric High Altitude Platform (HAP) could act as a relay station to forward the optical communication beam over the last $20 \mathrm{~km}$ through the atmosphere to the ground station, where short-range, high data-rate microwave systems are feasible. This paper will discuss the capabilities of HAP and GEO relay stations to increase the downlink capacities of LEO satellites. Environmental aspects for the deployment of HAP relays and regulatory/technology issues for a microwave downlink on the last $20 \mathrm{~km}$ to the ground will be discussed.
\end{abstract}

Keywords: High-Altitude Platform Relays, Optical Free-Space Communication, Satellite Downlinks

\section{INTRODUCTION}

Recent years have seen an immense increase in the capability of earth observation (EO) sensors flown on satellites. State-of-the-art payloads like high resolution optical/infrared cameras or SAR systems produce data at rates of several gigabits per second (Gbps). As a consequence conventional microwave (MW) downlinks have become the bottleneck in EO systems, as they are limited to some hundred megabits per second. The data acquired by the sensor can only be sent to the ground when it is in the reach of an according MW ground station antenna, which happens a few times per day with each downlink session lasting only around 9 minutes at maximum for a LEO (Low Earth Orbit) satellite. Due to the limited downlink capacity from LEO satellites the effective operational time for EO sensors is restricted to few minutes a day. The MW downlink technology is currently reaching its limits due to limitations in the available frequency bands but also in technological feasibility and therefore cannot be adapted accordingly.

To solve this communications bottleneck, i.e. to increase the effective downlink capacity, the use of optical free-space, high-bandwidth links is proposed. This technology instantly multiplies the downlink data rate by a factor of ten, while even faster links would be feasible in the near future deploying methods like Wavelength Division Multiplexing (WDM). At the same time the mass, size, and power consumption of the satellite terminals decrease to a fraction of the values of conventional MW-antennas. High speed downlinks would become an option even for compact- or micro-satellites (a typical Tx-aperture diameter would be approx. 3 centimeters). Furthermore, also the size of the according Optical Ground receiving Station (OGS) remains quite compact, with only some decimeters of telescope diameter, enabling transportable or even mobile stations. This is an important benefit compared to MW ground stations which typically have

Markus.Knapek@dlr.de; phone +49 8153 282879; www.dlr.de

Copyright 2007 Society of Photo-Optical Instrumentation Engineers. This paper was published in SPIE Free-Space Laser Communications VII and is made available as an electronic reprint with permission of SPIE. One print or electronic copy may be made for personal use only. Systematic or multiple reproduction, distribution to multiple locations via electronic or other means, duplication of any material in this paper for a fee or for commercial purposes, or modification of the content of the paper are prohibited. 
antenna diameters of 5 meters. The inherent tap-proofness of directed optical beams due to a minimized optical signal spot beam on ground (typically between 10 and $50 \mathrm{~m}$ ) is also very appreciable in security applications.

Optical links have been demonstrated in various scenarios over the last decade. Inter-satellite links were performed in Europe between the geostationary satellite Artemis and the LEO satellite Spot-4 on a semi-operational basis [1]. Downlinks were demonstrated from Artemis to the Optical Ground Station (OGS) on Tenerife [2], operated by ESA. An optical link from a stratospheric balloon platform at $22 \mathrm{~km}$ altitude was performed in Kiruna, Sweden, by the Institute of Communications and Navigation of the German Aerospace Center (DLR) in August 2005 [3][4]. In 2006 DLR has demonstrated together with JAXA the feasibility of direct optical LEO-downlinks in a link from the Japanese LEO satellite OICETS to the DLR OGS near Munich, Germany [5][6]. Several inter-satellite and downlink experiments are planned for end of 2007 from the LEO satellites TerraSAR-X and NFIRE with the German Laser Communication Terminal at $5.6 \mathrm{Gbps}$.

\section{HIGH ALTITUDE PLATFORMS AS RELAY STATIONS FOR DOWNLINKS FROM LEO SATELLITES}

Optical links offer significantly higher bandwidth, however they are blocked by clouds. As a consequence downlinks from satellites to a ground station have a limited availability depending on the cloud situation over a site. For non-EO applications like communications or broadcast, a nearly hundred percent availability is required for the satellite link. This problem can be solved by using a relay station in form of a High Altitude Platform (HAP) positioned above the clouds in about $20 \mathrm{~km}$ altitude [7]. An optical link from the satellite to the HAP would have a hundred percent availability, as it is not hindered by clouds. The final "last mile" to the ground could then be bridged by a standard MW point-to-point link as used today in terrestrial applications, but with a large bandwidth compared to a satellite link due to the short distance. An optical link in parallel to the MW link could increase the bandwidth of the last $20 \mathrm{~km}$ several times during cloud-free conditions.

Fig. 1 shows two scenarios for optical downlinks from LEO satellites. In the HAP relay scenario the data is sent directly via a HAP to the ground. In the scenario geostationary satellite (GEO)-HAP relay the data is first transmitted to a GEO satellite and then via a HAP to the ground. The link HAP-ground (HAP-GND) can be established by an optical link or alternatively by a MW link. A network of interconnected HAPs could provide almost full availability also for optical HAP-GND links.

Link duration between the LEO satellite and the HAP will be up to 12 minutes for each contact, with about 3-15 contacts depending on the geographic latitude of the HAP. In order to increase contact times a GEO satellite could be introduced. Link availability to the GEO satellite would be about half of the LEO orbit, i.e. about 12 hours per day. From the GEO satellite the data would be transmitted on a continuous link to the HAP. The increased link duration would be at the cost of significantly longer link distances with a more stringent linkbudget and the additional expenses of a GEO satellite.

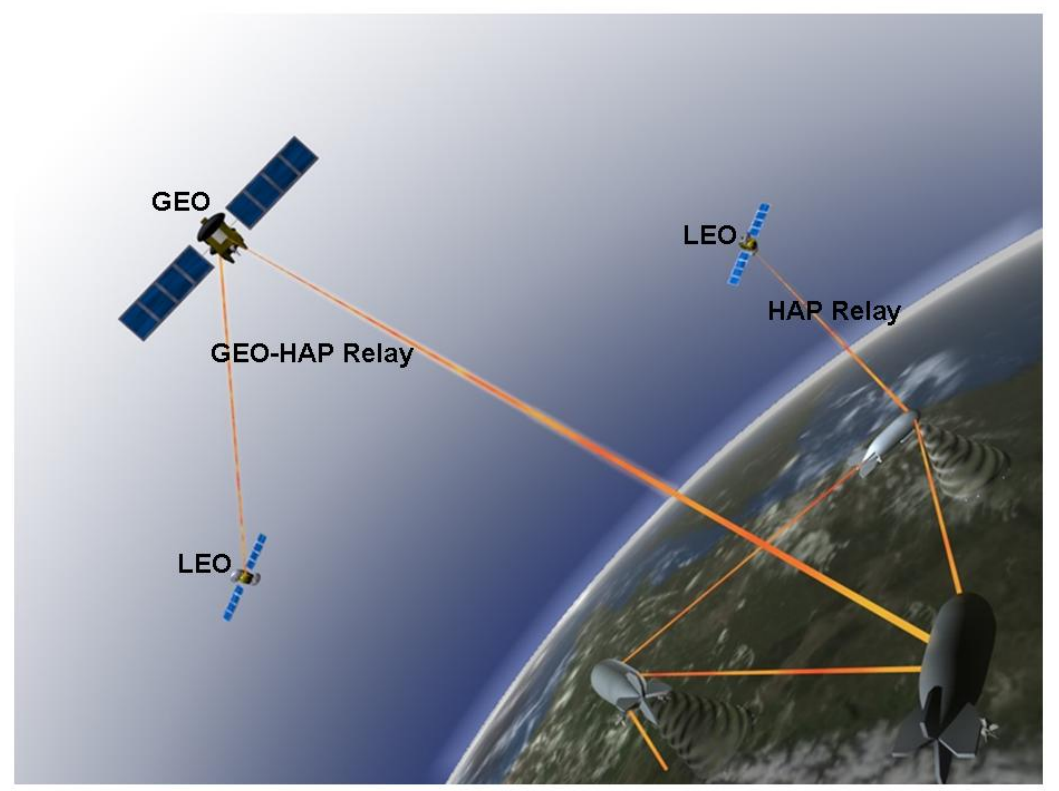

Fig. 1. Relay link from a LEO satellite to the ground via HAP relay (right) and from a LEO satellite over a GEO satellite and a HAP (left). The "last mile" downlink from the HAP to a ground station would be either bridged by a high-bandwidth optical or microwave link. 


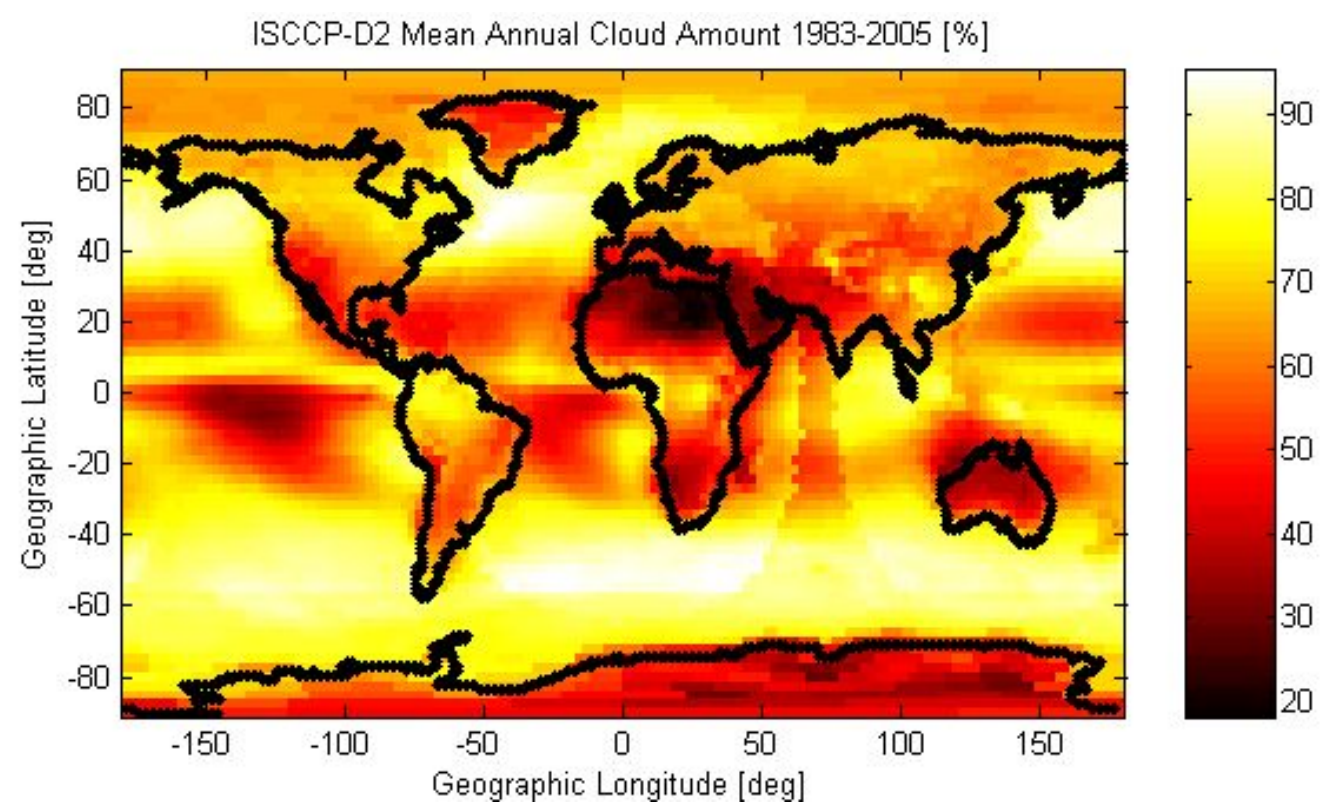

Fig. 2. Mean annual cloud amount for the years 1983-2005. Source ISCCP [10].

\section{OPTIMAL GEOGRAPHIC POSITIONING FOR HIGH ALTITUDE PLATFORMS}

\subsection{Availability of optical links to the ground taking cloud coverage into consideration}

Clouds are a crucial issue for optical links to the ground, as they in most cases block optical communication. Only very thin clouds can be handled with an adequate link margin. Fig. 2 shows the world's mean annual cloud cover for the years 1983-2005. Minimum values of cloud blockage appear at about 20 degrees latitude south and north with as low as $20 \%$. Significantly higher values are observed for moderate latitudes (e.g. Europe, USA) of up to $75 \%$. In order to find favourable locations for optical links to the ground, seasonal changes have to be taken into account. High resolution data on cloud coverage over Europe can be found at the World Data Center [8][9] and worldwide but with significantly lower resolution at the International Satellite Cloud Climatology Project [10]. In some cases mountain tops as for classical astronomical sites might offer more favourable cloud coverage, as they are located above low cloud layers.

\subsection{Stratospheric winds influencing the positioning accuracy of HAPs}

Wind speeds at the altitude of the platform are an important aspect of keeping a HAP geostationary, i.e. positioned at a certain geographic latitude, longitude, and height within a well-defined station-keeping box. The higher the wind speeds are the more energy will be necessary to fulfil the station-keeping requirements and the higher will be the stress to the structure of the HAP.

Fig. 3 shows zonal wind speeds (along latitude circle) in the atmosphere [11][12]. The figures are based on the results of a semi-empirical model, where three models where combined to get monthly wind averages from 0 - $100 \mathrm{~km}$ altitude. For lower altitudes up to $55 \mathrm{~km}$ reanalyzed Met Office data were used. For altitudes from $70-100 \mathrm{~km}$ the Global Empirical Wind Model (GEWM) was taken. The gap from 55-70km was filled by COMMA numerical model data. The data gives a good estimate of favourable heights and latitudes for HAP positions in respect to average wind speed, however the data does not allow a conclusion about peak wind velocities, as they are averaged out over each month. The shown model is based on data of the late $20^{\text {th }}$ century. Meridional wind speeds have much lower values and are not taken into consideration for this discussion. 

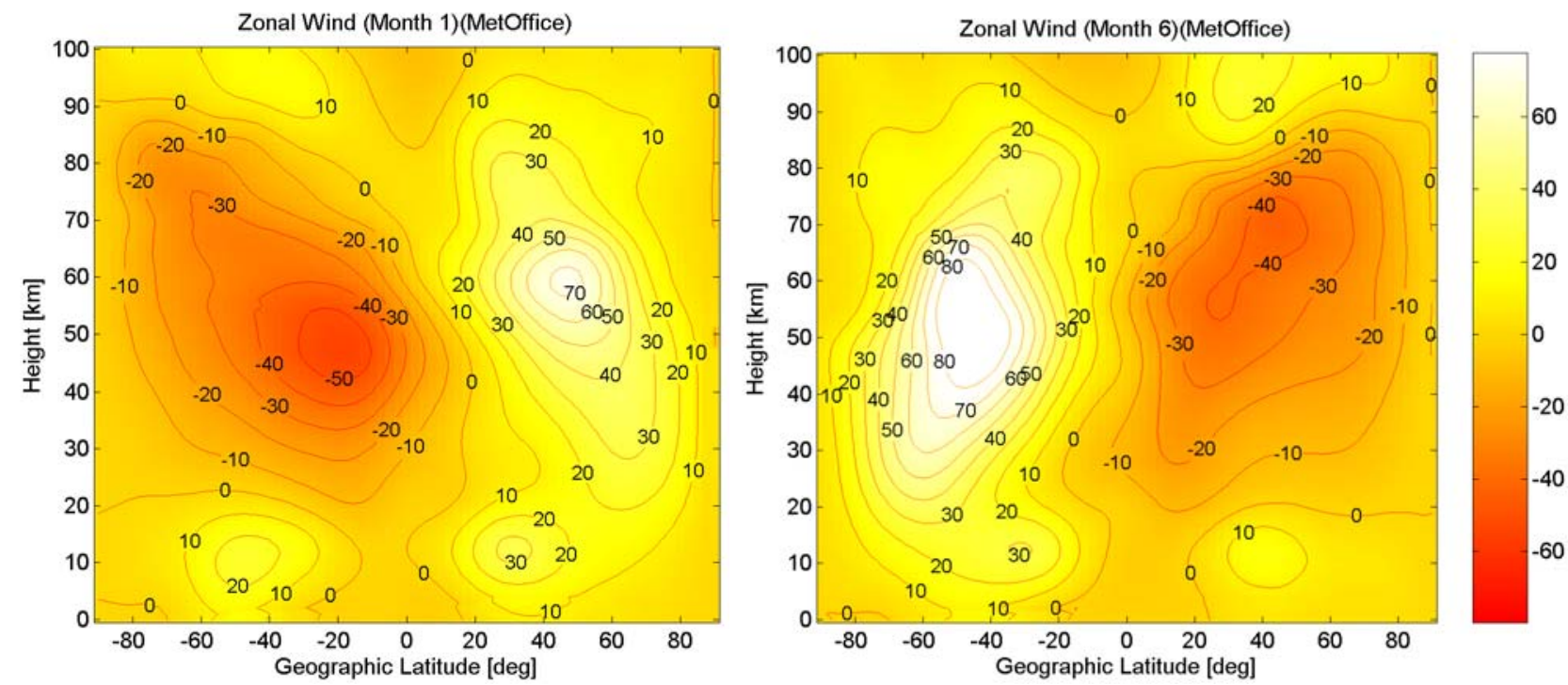

Fig. 3. Zonal wind speed [m/s] (along latitude circle) for January (left) and June (right). Meridional winds (along meridians) have much lower levels and can be neglected in comparison with zonal winds.

HAPs are planned for altitudes typically around 22km. Fig. 3 indicates favourably low wind speeds for these altitudes. Fig. 4 shows a detailed plot of the wind speeds at $22 \mathrm{~km}$ altitude over the geographic latitude. Data basis are 12 monthly data sets, which represent mean values of the whole measurement period. The 12 monthly data sets were averaged to give the annual mean values. The maximum value of the 12 data sets was taken to give the maximum values in Fig. 4. The absolute values for the wind speed were calculated, since only the wind speed is of importance and not the direction.

Especially strong winds can be observed for latitudes between $40-80^{\circ}$ south and north. These winds are caused by the polar vortex, a persistent large-scale cyclone located near the Earth's poles. The Antarctic polar vortex is more pronounced and persistent than the Arctic one; this is caused by the distribution of land masses at high latitudes in the northern hemisphere, which contributes to a breakdown of the Arctic vortex. The low wind speeds close to the equator appear in the graphs due to an averaging effect of a biennial oscillation of the wind direction; however wind speeds are quite large in these latitudes. Most favourable maximum (averaged) wind speeds appear at about $30^{\circ}$ latitude south and

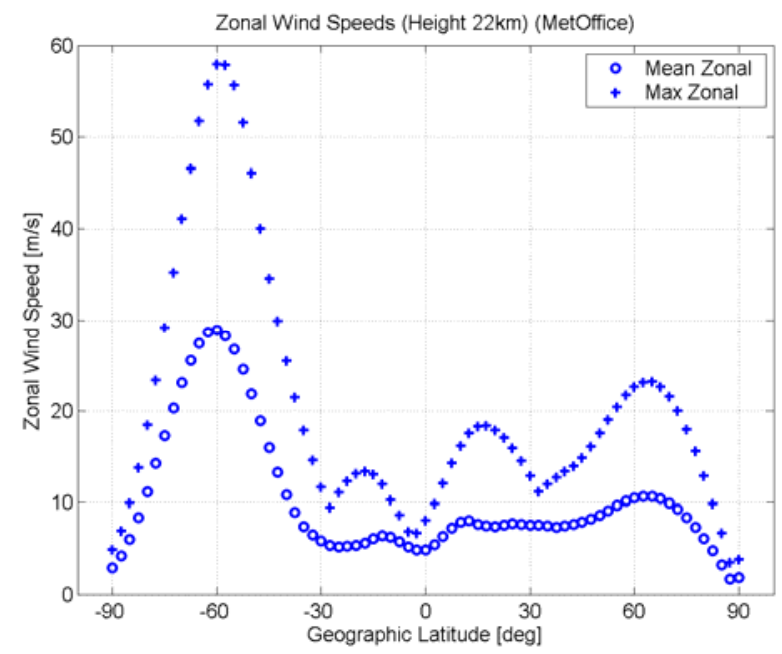

Fig. 4. Zonal winds (along latitude circle) at $22 \mathrm{~km}$ altitude. Mean values give the average (absolute) wind speed over the year. Maximum values give the maximum of the monthly values in the year. 


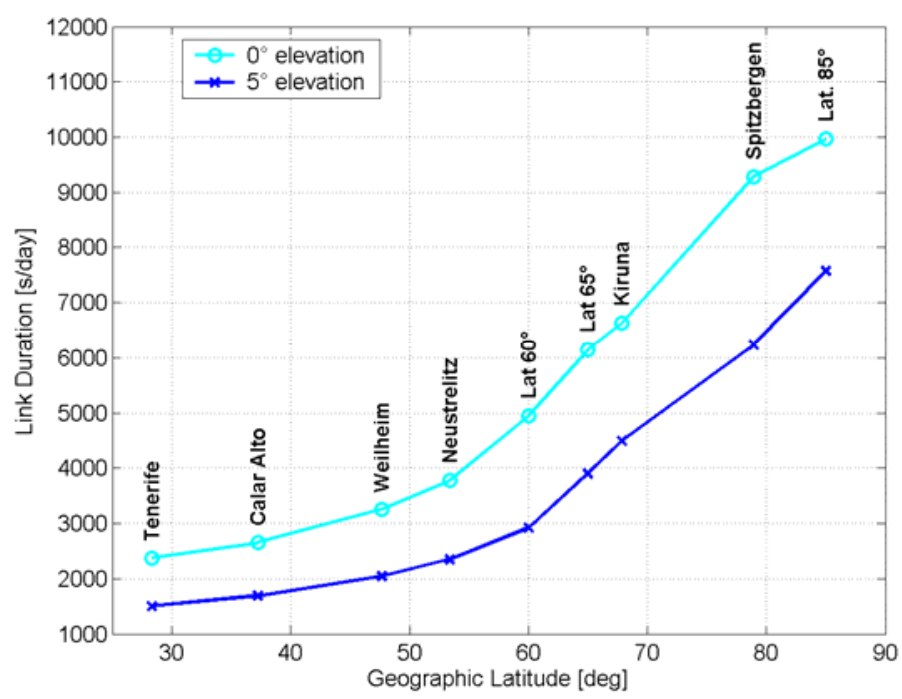

Fig. 5. Link duration from an earth-observation satellite (TerraSAR-X) to ground stations at various latitudes. Link durations for minimum elevation angles of zero and 5 degrees as a constraint are shown. Link durations slightly change for a LEO-HAP link with the HAP at $20 \mathrm{~km}$ altitude.

north, where the maximum wind speed stays over the year at around $11 \mathrm{~m} / \mathrm{s}$. Therefore positions for HAPs at $22 \mathrm{~km}$ altitude could be between $10-40^{\circ}$ north or $10-30^{\circ}$ south, with even lower wind speeds at about $33^{\circ}$ north and $28^{\circ}$ south.

\subsection{Link duration from the LEO satellite to the HAP}

The number of contacts and therefore the link duration per day from a LEO satellite to a HAP strongly depends on the geographic latitude of the HAP location and the orbit of the satellite. Fig. 5 shows link durations for TerraSAR-X as an example of a typical EO LEO satellite. TerraSAR-X has a nearly polar orbit with $97.4^{\circ}$ inclination and $508 \mathrm{~km}$ orbit height. The difference in the link duration is mainly caused by the contact number to the HAP. There are about 3 contacts per day for a HAP position at low latitudes and up to 15 close to the earth poles.

The link duration from a LEO satellite to the HAP or ground station also depends on the minimum elevation angle under which the satellite can be seen and a link is possible. For stations on the ground larger elevation angles $\left(5-10^{\circ}\right)$ are required to establish a communication link due to a limited horizontal line of sight and atmospheric turbulence/attenuation. For links to HAPs elevation angles even below the horizon $\left(-2^{\circ}\right)$ are possible due to the elevated position of the platforms. The maximum link duration per pass is about 8 minutes at a minimum angle of $10^{\circ}$ and 13 minutes for $-2^{\circ}$ elevation.

\section{CAPACITIES OF FREE-SPACE OPTICAL LINKS}

Free-space optical links have highly directive beams with very small divergence angles due to the short wavelength in the NIR. As a consequence optical systems are extremely power efficient over long distances and allow highest data rates. No regulatory issues exist for optical links and therefore capacities are not limited by the available spectrum.

For the selection of the transmission frequency mainly the availability of qualified components with sufficient output power for the chosen modulation format on the satellite is necessary. Two different modulation technologies are currently used for free-space optical communications. The coherent technology (e.g. BPSK) features higher sensitivity and nearly no degrading sensitivity with background radiation. A typical value for system sensitivity of existing systems is 40 Photons/bit $(-54 \mathrm{dBm})$ at data rates of $1 \mathrm{Gbps}$ and a wavelength of $1.064 \mu \mathrm{m}$ (BER $\left.10^{-6}\right)$. Comparable intensitymodulated systems with direct detection and APD photo detectors usually have sensitivities in the region of 140 Photons/bit $(-46 \mathrm{dBm})$ at $1.550 \mu \mathrm{m}$. State-of-the-art communication systems offer several Gbps transmission rate, e.g. 5.6 Gbps on the LEO satellite TerraSAR-X. 


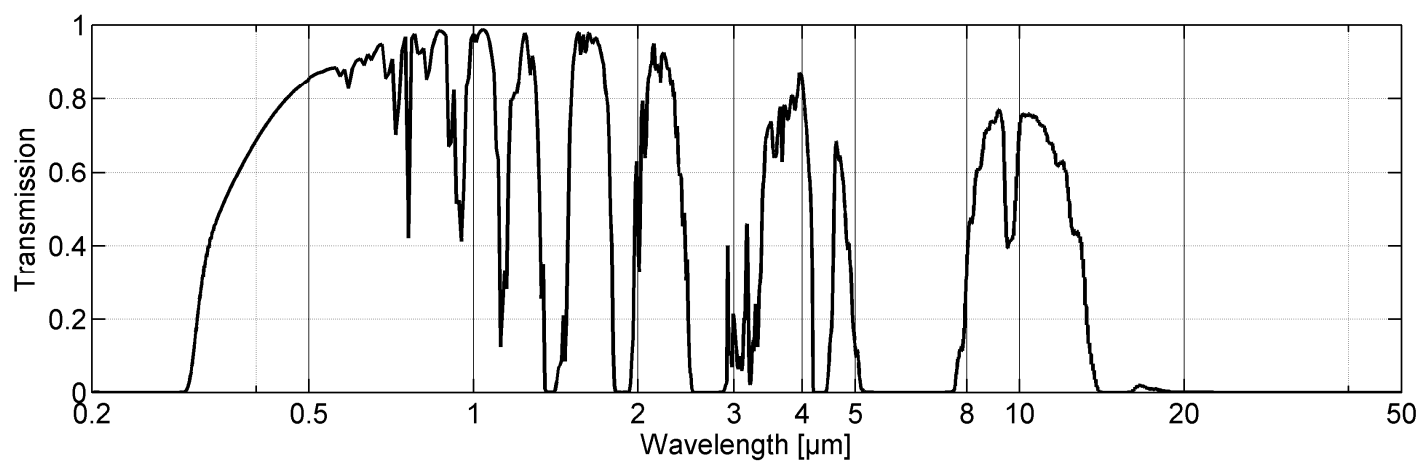

Fig. 6. Transmission windows through the atmosphere at optical wavelengths from the visible to the infrared.

For both wavelength and modulation formats high output powers are possible due to the availability of optical amplifiers. Whereas fiber amplifiers at $1.064 \mu \mathrm{m}$ are already qualified for LEO applications and about to be tested on TerraSAR-X, no usage of Erbium Doped Fiber Amplifiers (EDFA) for the optical C-band in space is known so far. In principle they are subjected to the same aging process [13].

An additional criterion for links through the atmosphere is the availability of atmospheric clear-air transmission windows. Fig. 6 shows the atmospheric transmission for downlinks to the ground from the visible to the mid-range infrared at $20 \mu \mathrm{m}$. Both wavelengths, $1.064 \mu \mathrm{m}$ and $1.55 \mu \mathrm{m}$, fall into good transmission windows. The attenuation for optical links from satellites to HAPs is almost negligible due to the altitude of the HAP at $22 \mathrm{~km}$ and stays below $1.5 \mathrm{~dB}$. For HAP-GND links clouds have a decisive influence. The use of other wavelengths has been studied, especially $10.6 \mu \mathrm{m}$ of $\mathrm{CO}_{2}$ laser, to improve the transmission through clouds [7], but with moderate results.

\section{CAPACITIES OF THE MICROWAVE HAP-GND LINK}

\subsection{Regulatory issues related to downlink transmission from earth observation satellites or High Altitude Platforms}

According to the Space Frequency Coordination Group [14] and the International Telecommunication Union [15], the frequency bands available for high data rate earth exploration applications for Earth Exploration Satellites (EES) are $8025-8400 \mathrm{MHz}$ and $25.25-27 \mathrm{GHz}$ for applications bearing unavailability in case of rain.

According to the European Radio Communication Committee [16], High Altitude Platforms (HAP) may use for downlink transmission the following frequency bands: 47,2-47,5 GHz and 47,9-48,2 $\mathrm{GHz}$.

\subsection{Typical technologies and bit rates in downlinks of classical EO satellites}

Previous studies carried out at DLR have shown that a LEO satellite (with circular orbit at $558 \mathrm{~km}$ altitude) for EO missions is seen from any ground station with a varying elevation angle, and for a limited amount of time during the day. The total daily satellite visibility time can be increased using several ground stations spread over the earth. This is unfortunately not always possible, especially for military applications, due to many geo-political issues.

Studies have shown that the earth observation satellite is seen during a considerable amount of time with a low elevation angle; this time should be profitably used to transfer traffic (images) from the satellite to the ground station. At Ka-band, nevertheless, high availability cannot be guaranteed at low elevation angle because of high attenuation due to rain (on the order of 50,25, 18, 13 and $6 \mathrm{~dB}$ attenuation at $10,20,30,45$ and $70^{\circ}$ elevation at $26 \mathrm{GHz}$ for a ground station located in Germany at $99,9 \%$ availability, according to [17]).

Due to the (rapid) variations of transmission conditions between the satellite and any ground station (resulting from the variation of the elevation angle and of the different propagation conditions), Adaptive Coding and Modulation (ACM) 
techniques may be used for downlink transmission between the satellite and the ground station to optimize the volume of data transmitted per unit of time.

This gain, as well as typical transfer capacities of a LEO satellite system in X band $(8.2 \mathrm{GHz}, 300 \mathrm{MHz}$ bandwidth, 295 $648 \mathrm{Mbps}$ ), is illustrated in the following table when the antenna of the satellite is a single fixed reflector, the total satellite transmission power is $58 \mathrm{~W}$, the considered availability $99.9 \%$ and the ground station is supposed to be located in the north east of Germany:

Table 1: Sidereal day transmission capacity comparison for an EO satellite dynamically using different coding and modulation schemes (ModCod 1-3, 295-648Mbps) according to the transmission conditions.

\begin{tabular}{|c|c|c|c|c|c|}
\hline & Selected ModCod $^{1}$ & 1 & 2 & 3 & \\
\hline $\begin{array}{l}\text { Mod Cod } \\
\text { Combination }\end{array}$ & ModCod Throughput & $0,295 \mathrm{Gbps}$ & $0,54 \mathrm{Gbps}$ & $0,648 \mathrm{Gbps}$ & $\begin{array}{l}\text { Total Transmitted Capacity } \\
\left(\text { Gbit/sidereal day }{ }^{2}\right)\end{array}$ \\
\hline Only 1 & ModCod Duration & $2257,8 \mathrm{~s}$ & $0 \mathrm{~s}$ & $0 \mathrm{~s}$ & 666,05 \\
\hline Only 2 & ModCod Duration & $0 \mathrm{~s}$ & $1472,8 \mathrm{~s}$ & $0 \mathrm{~s}$ & 795,31 \\
\hline Only 3 & ModCod Duration & $0 \mathrm{~s}$ & $0 \mathrm{~s}$ & $927,06 \mathrm{~s}$ & 600,73 \\
\hline $1+2$ & ModCod Duration & $785 \mathrm{~s}$ & $1472,8 \mathrm{~s}$ & $0 \mathrm{~s}$ & 1026,89 \\
\hline $1+3$ & ModCod Duration & $1330,74 \mathrm{~s}$ & $0 \mathrm{~s}$ & $927,06 \mathrm{~s}$ & 993,3 \\
\hline $1+2+3$ & ModCod Duration & $785 s$ & $545,74 \mathrm{~s}$ & $927,06 \mathrm{~s}$ & 1127 \\
\hline Clear Sky ${ }^{3}$ & ModCod Duration & $0 \mathrm{~s}$ & $0 \mathrm{~s}$ & $2246,6 s$ & 1455,8 \\
\hline
\end{tabular}

A Ka-Band downlink is also possible, but simulation results have shown that for the same availability only a lower capacity can be reached per day because of severe attenuation due to rain, especially at low elevation angles. Therefore, LEO satellites for EO with a low number of downlink earth stations should preferably work at X-band [18][19][20][21], preferably with ACM (more than 1000 Gbit of data per sidereal day can be transmitted from the LEO satellite to a ground station in this case).

\section{3. “Last Mile” microwave HAP-GND link}

The use of a HAP relay station above a ground station brings a number of advantages for a MW HAP-GND link:

- Quasi-stationary HAP flying over the ground station and guaranteeing that the ground station always looks towards the HAP with a high elevation angle and therefore reduced atmospheric/rain attenuation.

- Reducing the HAP-GND distance in comparison with the LEO-GND distance, considerably decreasing the freespace losses and therefore greatly improving the link budget of the MW link.

- HAPs can carry a heavy telecommunication payload with high power and efficient technology available on-board (e.g. high gain narrow beam steering antenna tracking the ground station), which helps to improve the link budget.

- Large storage capacities can be foreseen in the payload of the HAP, as radiation is not an issue for these altitudes. In the following, the link budget of the downlink MW link between the HAP and the ground station is presented for a Vband $(48 \mathrm{GHz})$ system requiring high availability $(99,9 \%)$, with a ground station illustratively located in Neustrelitz (latitude $=53.35^{\circ}$, longitude $=13.05^{\circ}$ ), a ground station widely used in Germany. In order to simulate a worst case, a low HAP transmission power (10W) as well as a tapered aperture antenna with low maximum gain (8.3 dB) has been selected. Under these hypotheses, a simple payload architecture for the HAP can be envisaged. The link budget could be

\footnotetext{
${ }^{1}$ Different ModCod (Modulation and Coding scheme) are dynamically selected according to the transmission conditions: ModCod 1: QPSK modulation, convolutional code, rate 2/3, concatenated with shortened RS code, rate 188/204,

ModCod 2: 8PSK modulation, 3/4 Coding rate (DVB-S2 like, $\mathrm{BCH}+\mathrm{LDPC}$ ),

ModCod 3: 8PSK modulation, 9/10 Coding rate (DVB-S2 like, BCH + LDPC),

${ }^{2}$ A sidereal day corresponds to 86164 seconds.

${ }^{3}$ For clear sky conditions, it has been assumed that there is neither rain nor gaseous attenuation at any possible elevation angle.
} 
improved by increasing the transmission power and using a steering antenna with high transmission gain, but as seen in the following table this is not necessary. The Ka-band ground station and antenna is similar to the ViaSat 8073 Ka-band earth station antenna [22], using a $7.3 \mathrm{~m}$ dish.

A bandwidth of $300 \mathrm{MHz}$ with 32-APSK modulation and 9/10 BCH + LDPC code rate has been selected, as specified in DVB-S2 [23].

Table 2: Linkbudget for the MW link HAP-GND over the last $20 \mathrm{~km}$. The HAP station would be located close to the zenith above the ground station, so only large elevation angles with low attenuations have to be considered.

\begin{tabular}{|l|l|l|l|}
\hline Elevation angle $\left(^{\circ}\right)$ & $60^{\circ}$ & $70^{\circ}$ & $80^{\circ}$ \\
\hline Transmision Power $(\mathrm{dBW})$ & 10 & 10 & 10 \\
\hline HAP Feeder Losses $(\mathrm{dB})$ & 2 & 2 & 2 \\
\hline HAP Antenna Gain $(\mathrm{dB})$ & 6,7 & 7,6 & 8,1 \\
\hline HAP EIRP $(\mathrm{dBW})$ & 14,7 & 15,6 & 16,1 \\
\hline Free Space Loss $(\mathrm{dB})$ & 154,13 & 153,42 & 153,0 \\
\hline $\begin{array}{l}\text { Rain Attenuation } \\
(99,9 \% \text { availability, dB) }\end{array}$ & 23,6 & 13,31 & 12,67 \\
\hline $\begin{array}{l}\text { Gazeous Attenuation } \\
(99,9 \% \text { availability, dB) }\end{array}$ & 1,21 & 1,12 & 1,07 \\
\hline Polarization mismatch losses $(\mathrm{dB})$ & 0,3 & 0,3 & 0,3 \\
\hline Flux at ground station $\left(\mathrm{dBWm}{ }^{-2}\right)$ & $-164,6$ & $-152,5$ & $-151,0$ \\
\hline Gain reception antenna $(\mathrm{dB})^{6}$ & 69,07 & 69,07 & 69,07 \\
\hline Antenna Temperature $(\mathrm{K})$ & 448 & 437 & 435 \\
\hline G/T $(\mathrm{dB} / \mathrm{K})$ & 42,55 & 42,66 & 42,69 \\
\hline Antenna Reception pointing losses $(\mathrm{dB})$ & 1,33 & 1,33 & 1,33 \\
\hline C/N0 $(\mathrm{dBHz})$ & 105,9 & 118 & 119,6 \\
\hline Eb/N0 (dB) & 15,5 & 27,6 & 29,3 \\
\hline Eb/N0 required @ $10^{-6}$ BER (dB) & 9,6 & 9,6 & 9,6 \\
\hline
\end{tabular}

Under these hypotheses and according to the DVB-S2 standard, transmission could occur in $99.9 \%$ of the time with a bit rate of $300 \mathrm{MHz} \cdot 4.453 \frac{\mathrm{bit}}{\mathrm{Hz}}=1336 \mathrm{Mbps}$, where $4.453 \frac{\mathrm{bit}}{\mathrm{Hz}}$ gives the spectral efficiency for 32APSK modulation and $9 / 10$ $\mathrm{BCH}+\mathrm{LDC}$ code rate.

Nevertheless, this corresponds to a maximum theoretical limit, and hardware enabling to reach such a high bitrate does not exist yet. Current of the shelf equipment [18][25][26] achieves up to $800 \mathrm{Mbps}$ in X-Band, which can be upconverted to reach the desired V-band frequency for a downlink transmission from the HAP. Therefore, with the considered hybrid satellite/HAP system using optical transmission between the satellite and the HAP, and MW transmission between the HAP and the ground station, a continuous stream of up to $800 \mathrm{Mbps}$ can be transmitted to the ground station.

\section{COMPARISON OF DOWNLINK THROUGHPUT}

The link capacity (e.g bits per day) from the LEO satellite to the ground depends on several factors:

- Downlink bitrate (of the segment with the lowest capacity) in bits per second. Modern optical systems like the LCT on TerraSAR-X have bitrates of $5.6 \mathrm{Gbps}$ over several $1000 \mathrm{~km}$ and $2.8 \mathrm{Gbps}$ for GEO distances $(40000 \mathrm{~km})$. MW links operate at several 100Mbps. Wavelength Division Multiplex WDM methods are envisioned for optical links, which would significantly increase link capacities into the $100 \mathrm{Gbps}$ region.

- Cumulative link duration per day (line of sight). The link duration for links from LEO satellites to HAPs or ground stations mainly depends on the number of satellite passes over a station and the minimum elevation angle.

\footnotetext{
${ }^{4}$ According to the ITU-R P model [17].

${ }^{5}$ According to the ITU-R P model [24].

${ }^{6}$ Gain of a $7.3 \mathrm{~m}$ diameter dish at $48 \mathrm{GHz}$.
} 
- Cloud-free time is a crucial issue for optical links to the ground. Minimum values appear at about 20 degrees latitude north and south.

Table 3 shows the link parameters for 5 exemplary scenarios. Scenario 1-3 use only a HAP as relay station. Scenarios 4 and 5 include an additional GEO satellite. 5.6Gbps were assumed for LEO-HAP optical (OPT) links (Scenario 1,4), 2.8Gbps for LEO-GEO/GEO-HAP optical links (Scenario 4,5), and 800Mbps for MW links HAP-GND (Scenario 2,3,5). A minimum elevation angle of zero degrees was taken to establish a link LEO-HAP.

- Scenario 1: LEO-HAP-GND with an optical link HAP-GND considering cloud coverage. No data buffered on the HAP.

- Scenario 2: LEO-HAP-GND with a MW link HAP-GND with full availability. No data buffering on the HAP.

- Scenario 3: Same as 2, but data buffering on the HAP and therefore 5.6Gbps over the whole chain LEO-HAPGND.

- Scenario 4: LEO-GEO-HAP-GND with an optical link HAP-GND considering cloud coverage. No data buffering on the HAP.

- Scenario 5: LEO-GEO-HAP-GND with a MW link HAP-GND with full availability. No data buffering on the HAP.

The transferable mean amount of data per day $D$ for an optical HAP-GND link is calculated from the effective data rate $f_{\text {eff; }}$, the link duration $T$ per day in dependence of the geographic latitude $\lambda$ and the minimum elevation angle $\alpha$, and the probability of cloud blockage, which depends mainly on the latitude $\lambda$ and the time of the year $t$.

$$
D=f_{\text {eff }} \cdot T(\lambda, \alpha) \cdot\left[1-p_{\text {clouds }}(\lambda, t)\right]
$$

Similar formulas apply for the other scenarios. Data transmission is given for one satellite and for full utilization, if several satellites utilize the full capacity of the relay HAP. Data buffering on the HAP can be employed when the MW HAP-GND link has less capacity than the optical LEO-HAP link. Data transmission would continue from the buffer when the satellite would be already out of sight. Data transfer to the ground would be delayed but the full optical link capacity could be used. Data delay would be acceptable for EO missions, however not for real-time communication.

A future network of HAP interconnected with optical links would eliminate the cloud blockage problem of optical links by providing optical HAP-GND links at different geographic locations. HAP constellations in favourable locations achieve an overall link availability of over 95\% [8][27]. In addition the HAP network would multiply the link duration LEO-HAP by the number of HAPs and therefore the transferable data volume.

Table 3: List of the different scenarios with details about the links to the relay stations (HAP/GEO).

\begin{tabular}{|c|c|c|c|c|c|c|}
\hline Scenario & $\begin{array}{c}1 \\
\text { LEO-HAP-GND }\end{array}$ & $\begin{array}{c}2 \\
\text { LEO-HAP-GND }\end{array}$ & $\begin{array}{c}3 \\
\begin{array}{c}3 \\
\text { LEO-HAP-GND } \\
\text { buffered }\end{array}\end{array}$ & $\begin{array}{c}4 \\
\text { LEO-GEO-HAP- } \\
\text { GND }\end{array}$ & $\begin{array}{c}5 \\
\text { LEO-GEO-HAP- } \\
\text { GND }\end{array}$ & \\
\hline Link to GND & OPT & $\mathrm{MW}$ & MW & OPT & MW & \\
\hline LEO-HAP & 5,6 & 5,6 & 5,6 & & & Gbps \\
\hline LEO-GEO & & & & 2,8 & 2,8 & Gbps \\
\hline GEO-HAP & & & & 2,8 & 2,8 & Gbps \\
\hline HAP-GND & 5,6 & 0.8 & 0,8 & 5,6 & 0,8 & Gbps \\
\hline Effective Data Rate & 5,6 & 0,8 & 5,6 buffered & 2,8 & 0,8 & Gbps \\
\hline Typ. Link Duration & $40-166$ & $40-166$ & $40-166$ & 720 & 720 & min./day \\
\hline Cloud Coverage (blockage) & $37-80$ & NA & NA & $37-80$ & NA & Percent \\
\hline $\begin{array}{l}\text { Data Transmission per Day for } \\
\text { One Satellite }\end{array}$ & 7.3-14.0 & $1,9-8,0$ & $13,3-55,8$ & $24.2-76.6$ & 34,6 & Tbit/day \\
\hline $\begin{array}{l}\text { Data Transmission per Day Full } \\
\text { Utilization }\end{array}$ & $96.8-306.3$ & 69.1 & 69,1 & 48.4-153.1 & 69.1 & Tbit/day \\
\hline
\end{tabular}

Optical Link

Microwave Link

Fig. 7 depicts the dependence of the link capacity for a single satellite (not full utilization) over the position of the HAP in geographic latitude for different scenarios. For the LEO-HAP-GND scenarios (Scenario 1-3) the link capacity increases at higher latitudes due to the increased number of links LEO-HAP, even despite the less favorable cloud 


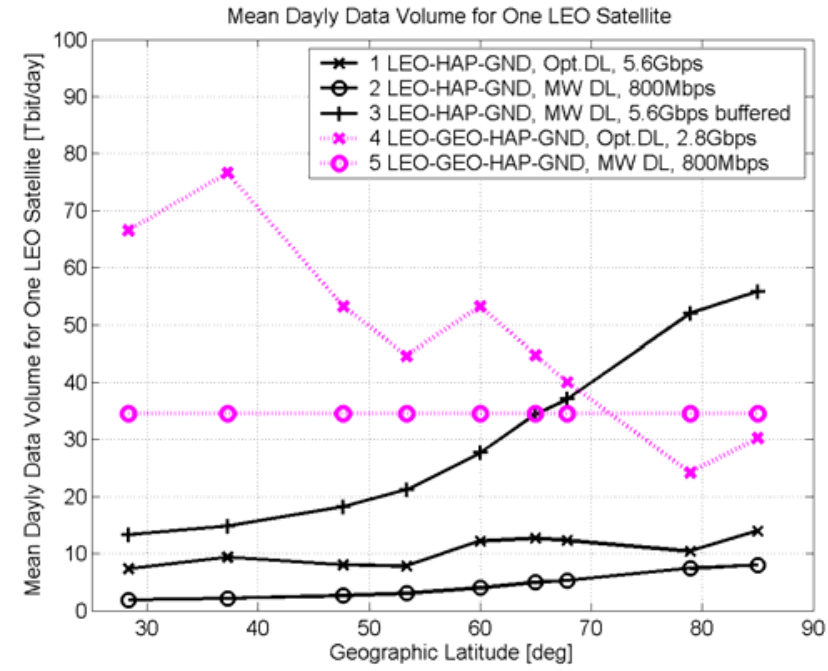

Fig. 7. Link capacity to the ground for a single LEO satellite in the various scenarios. Links including GEO satellites are marked with dashed lines.

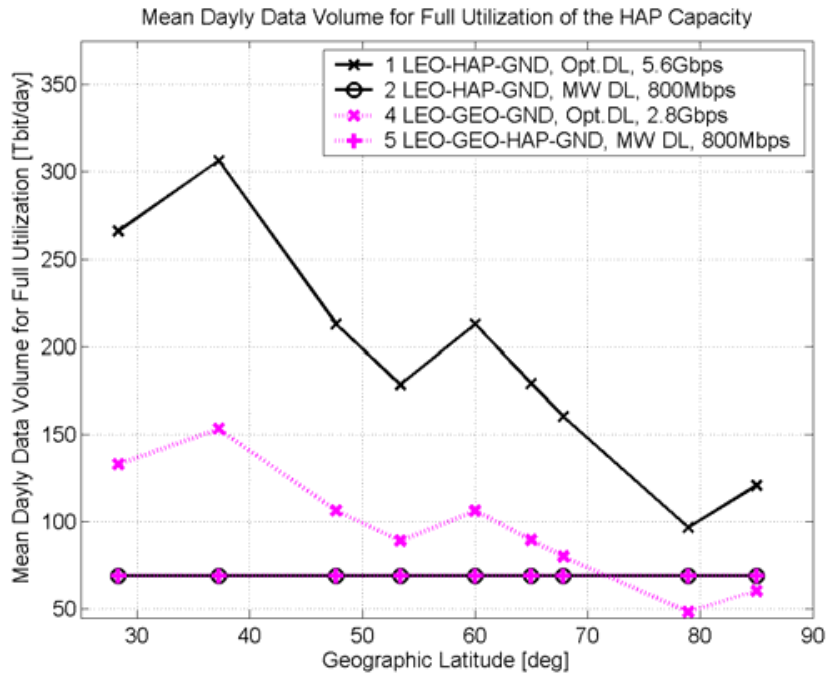

Fig. 8. Link capacity to the ground for full utilization of the HAP relay station. The buffered scenario is not applicable in the case of full utilization.

conditions at higher latitudes. For the GEO satellite scenario (Scenario 4) with an optical ground link only the effect of the cloud coverage remains. Link time would be constant at about 12 hours a day.

Fig. 8 shows the link capacities for full utilization of the HAP, i.e. when the HAP constantly transmits (100\% of time) the data of several LEOs to the ground. The cumulative data rates for all satellites are given. Rates decrease with the latitude for optical HAP-GND links (Scenario 1,4) due to cloud coverage.

The scenarios including GEO satellites (Scenario 4,5) have higher data rates for single LEO satellites (less than full utilization). For full utilization the HAP only scenarios give higher rates, which is due to the lower distances involved compared to the GEO distances. Longer distances reduce the available data rates for optical as well as for MW links. Important to mention is, that a GEO satellite can only serve on average 2 LEO satellites using the long link durations for GEO relays. A direct link LEO-HAP can serve on average about 10 satellites to utilize the full capacity of the HAP.

A typical X-Band LEO-GND link as on TerraSAR-X offers a data rate of 300Mbps. This results in a downlink capacity of about $0.45 \mathrm{~Tb}$ to $2.25 \mathrm{~Tb}$ per day $(1500 \mathrm{~s}-7500 \mathrm{~s}$ link duration per day in dependence of the latitude at a minimum elevation of $5 \mathrm{deg}$ ). In comparison the link via a HAP relay increases the downlink rate to 1.9-8.0 Tb per day (unbuffered) and 13.3-55.8Tb per day (buffered) (Scenario 2,3), both with full availability due to the MW link on the HAP-GND link. Typical link durations for LEO-HAP links are 2400-10000s at a minimum elevation angle of zero degrees elevation. Optical HAP-GND links can slightly increase downlink rates, but the link might be blocked for longer periods due to clouds. Optical HAP-GND links appear to be sensible for a HAP network of 3 or more stations, where the overall availability is close to $100 \%$. Also hybrid systems with an optical and in parallel a MW system provide full availability and higher data rates.

\section{DISCUSSION}

This paper has investigated the deployment of HAPs and GEO satellites as relay stations to improve the data transmission from LEO satellites to the ground. All investigated scenarios with HAP and GEO satellite relays with optical and MW links to the ground significantly increase the achieved data rates to the ground. Optical links were assumed for all inter-platform links, i.e. inter-satellite and satellite-HAP. The data rates achieved with a HAP relay can be increased by about a factor 30 compared to a typical MW downlink capacity at 300Mbps (e.g. TerraSAR-X) from a LEO satellite, even if only a high-bandwidth MW link HAP-GND is assumed with full availability. If optical links are considered to the ground, the link capacity can be further increased, but with limited availability or the need to solve the problem of cloud blockage (parallel MW link, network of HAPs). 
Optical data transmission from LEO satellites using GEO relay satellites have the advantage of higher link durations as they cover nearly half of the LEO orbit, however, the overall system complexity significantly increases with the requirement of an additional GEO satellite. Due to the larger link distances the terminal size, power consumption and weight of optical LEO-GEO link terminals are higher, which beyond that prevents the use of these terminals on small LEO satellites. The larger distances also decrease the achievable data rates. The financial effort to set up a GEO relay in the consequence becomes much higher than for scenarios without GEO satellites.

\section{REFERENCES}

1. T. T. Nielsen and G. Oppenhaeuser, "In orbit test result of an operational intersatellite link between ARTEMIS and SPOT4," Proc. SPIE, 4635, pp. 1-15, 2002.

2. A. Alonso, M. Reyes, and Z. Sodnik, "Performance of satellite-to-ground communications link between ARTEMIS and the Optical Ground Station", Proc. SPIE, Optics in Atmospheric Propagation and Adaptive Systems VII, 5572 , pp. 372-383, 2004.

3. J. Horwath, M. Knapek, B. Epple, M. Brechtelsbauer, and B. Wilkerson, "Broadband backhaul communication for stratospheric platforms: the Stratospheric Optical Payload Experiment (STROPEX)," Proc. SPIE, Free-Space Laser Communications VI, 6304, San Diego, 2006.

4. M. Knapek, J. Horwath, N. Perlot and B. Wilkerson, "The DLR ground station in the Optical Payload Experiment (STROPEX) - Results of the atmospheric measurement instruments", Proc. SPIE, Free-Space Laser Communications VI, 6304, San Diego, 2006.

5. Y. Takayama, T. Jono, M. Toyoshima, H. Kunimori, D. Giggenbach, N. Perlot, M. Knapek, K. Shiratama, J. Abe, and K. Arai, "Tracking and pointing characteristics of OICETS optical terminal in communication demonstrations with ground stations," Proc. SPIE Photonics West, Free-Space Laser Communication Technologies XIX, San Jose, Jan. 2007.

6. N. Perlot, M. Knapek, D. Giggenbach, J. Horwath, M. Brechtelsbauer, Y. Takayama, and T. Jono, "Results of the optical downlink experiment KIODO from OICETS satellite to Optical Ground Station Oberpfaffenhofen (OGSOP)," Proc. SPIE Photonics West, Free-Space Laser Communication Technologies XIX, San Jose, Jan. 2007.

7. D. Giggenbach, J. Horwath, and B. Epple, "Optical satellite downlinks to optical ground stations and high-altitude platforms," $16^{\text {th }}$ IST Mobile \& Wireless Communications Summit, Budapest, 2007.

8. F. Moll and M. Knapek, "Wavelength selection criteria and link availability due to Cloud Coverage Statistics affecting Satellite, Aerial, and Downlink Scenarios," to be published at the SPIE Free-Space Laser Communications VII, San Diego, Aug. 2007.

9. The World Data Center for Remote Sensing of the Atmosphere. Cloud Data: http://wdc.dlr.de/apollo/index.html

10. International Satellite Cloud Climatology Project (ISCCP), http://isccp.giss.nasa.gov/

11. C. Jacobi, K. Fröhlich, Y. Portnyagin, E. Merzlyakov, T. Solovjova, N. Makarov, D. Rees, A. Fahrutdinova, V. Guryanov, D. Fedorov, D. Korotyshkin, J. Forbes, A. Pogoreltsev, and D. Kürschner, "Semi-empirical model of middle atmosphere wind from the ground to the lower thermosphere," Submitted to Adv. Space Res.

12. Semi-Empirical Model of Middle Atmosphere Wind Data: http://www.uni-leipzig.de/ jacobi/intas03/data.htm.

13. M. Ott, "Radiation effects expected for fiber laser/amplifier rare earth doped optical fiber," Survey Report, http://misspiggy.gsfc.nasa.gov/photonics/) March 2004.

14. Space Frequency Coordination Group, http://www.sfcgonline.org/.

15. ITU-R SA.1024-1, "Necessary bandwidths and preferred frequency bands for data transmission from earth exploration satellites (not including meteorological satellites)", June 1997.

16. The European table of frequency allocations and utilisations covering the frequency range $9 \mathrm{kHz}$ to $275 \mathrm{GHz}$, ERCCEPT, ERC report 25, http://www.ero.dk/eca-change.

17. ITU-R P.618-8, "Propagation data and prediction methods required for the design of Earth-space telecommunication systems," April 2003.

18. CCSDS list of compliant equipments: http://public.ccsds.org/sites/databases/Lists/CCSDSProducts/AllItems.aspx.

19. IKONOS, http://directory.eoportal.org/pres_Ikonos2Block1.html.

20. IKONOS $X$ band Ground Station Specification, http://www.viasat.com/_files/_08fe203b613bc02b87de181a370e2bdf/pdf/1356mProductDescription.pdf.

21. J. Schwarz, H. Damerow, H. Maass, and J. Richter, "TerraSAR-X Ground Station at DLR in Neustrelitz," European Telemetry Conference, etc2004, Garmisch-Partenkirchen, May 24. - 27. 2004.

22. ViaSat Model 8073 Ka-Band Earth Station Antenna, http://www.viasat.com. 
23. Draft ETSI EN 302 307, "Digital Video Broadcasting (DVB); Second generation framing structure, channel coding and modulation systems for Broadcasting, Interactive Services, News Gathering and other broadband satellite applications," version 1.1.1, June 2004.

24. ITU-R P.676-5, "Attenuation by atmospheric gases," February 2001.

25. Thales Alenia Space on-board equipment, section payload/observation microwave: http://www1.alcatellucent.com/space/equipment/payload/obsmicro/index.htm.

26. L3 Communication Telemetry West, section Airborne Telemetry, http://www.l3 com.com/tw/telemetry_west/products/.

27. D. Giggenbach, R. Purvinskis, M. Werner, and M. Holzbock, "Stratospheric Optical Inter-Platform Links for High Altitude Platforms," Proc. of the 20th International Communications Satellite Systems Conference, Montreal, May 2002. 